This is a preprint of an article accepted for publication in the Journal of Combinatorial Designs (c) 2009 (copyright owner as specified in the journal). 


\title{
Biembeddings of Abelian groups
}

\author{
M. J. Grannell \\ Department of Mathematics and Statistics \\ The Open University \\ Walton Hall \\ Milton Keynes MK7 6AA \\ UNITED KINGDOM \\ (m.j.grannell@open.ac.uk) \\ M. Knor \\ Department of Mathematics \\ Faculty of Civil Engineering \\ Slovak University of Technology \\ Radlinského 11 \\ 81368 Bratislava \\ SLOVAKIA \\ (knor@math.sk)
}

July 2, 2009

\begin{abstract}
We prove that, with the single exception of the 2-group $C_{2}^{2}$, the Cayley table of each Abelian group appears in a face 2-colourable triangular embedding of a complete regular tripartite graph in an orientable surface.
\end{abstract}

\section{Running head:}

Biembeddings of Abelian groups

\section{AMS classifications: \\ 05B15, 05C10.}

\section{Keywords:}

Topological embedding, Latin square, Cayley table, Abelian group, complete tripartite graph. 


\section{Background}

A biembedding of two Latin squares of order $n$ is equivalent to a face 2-colourable triangular embedding of a complete regular tripartite graph $K_{n, n, n}$ in which the faces of each colour class generate the Latin squares. In this paper we determine which Latin squares formed from the Cayley tables of Abelian groups appear in such biembeddings.

In [4] a recursive construction was presented for biembeddings of Latin squares. This construction was used in that paper to prove that, for $i \neq 2$, the Cayley table of the Abelian 2-group $C_{2}^{i}$ appears in a biembedding. It was also conjectured that, with the single exception of the group $C_{2}^{2}$, the Cayley table of each Abelian group appears in a biembedding. In the current paper we give a proof of this conjecture. The difficulty in proving the result is due to the facts that the Cayley table of $C_{2}^{2}$ appears in no biembedding, while the unique biembeddings in which the Cayley tables of $C_{2}$ and $C_{4}$ appear are with copies of themselves, and both these squares lack transversals.

For general background material on topological embeddings, we refer the reader to [5] and [6]. Our embeddings will always be 2-cell embeddings in closed connected 2-manifolds without a boundary. A graph embedding is face 2-colourable if the faces may be coloured in such a way that any two faces with a common boundary edge receive different colours. It was shown in [2] that a triangular embedding of $K_{n, n, n}$ is face 2-colourable if and only if the supporting surface is orientable, and the surface is therefore a sphere with an appropriate number of handles.

A face 2-colourable triangular embedding of $K_{n, n, n}$ determines two transversal designs, $\operatorname{TD}(3, n)$, one for each colour class. Such a design comprises an ordered triple $(V, \mathcal{G}, \mathcal{B})$, where $V$ is a $3 n$-element set (the points), $\mathcal{G}$ is a partition of $V$ into three disjoint sets (the groups) each of cardinality $n$, and $\mathcal{B}$ is a set of 3-element subsets of $V$ (the triples), such that every unordered pair of elements from $V$ is either contained in precisely one triple or one group, but not both. The vertices of the embedded graph $K_{n, n, n}$ form the points of each design, the tripartition determines the groups, and the faces in each colour class form the triples of each design.

The connection with Latin squares is that a $\operatorname{TD}(3, n)$ determines a Latin square of order $n$ by assigning the three groups of the design as labels for the rows, columns and entries (in any one of six possible orders) of the Latin square. Conversely any Latin square of order $n$ determines a $\operatorname{TD}(3, n)$. Two Latin squares are said to be in the same main class or paratopic if the corresponding $\operatorname{TD}(3, n) \mathrm{s}$ are isomorphic. Thus a face 2-colourable triangular embedding of $K_{n, n, n}$ may be considered as a biembedding of two $\operatorname{TD}(3, n)$ s or, equivalently, two Latin squares. To be precise, we say that two Latin squares of order $n$ are biembeddable in a surface if there is a face 2-colourable triangular embedding of $K_{n, n, n}$ in which the face sets forming the two colour classes give paratopic copies of the two squares. 
Given a Latin square $L$ of order $n$, we may use the notation $k=L(i, j)$ to denote that entry $k$ appears in row $i$ column $j$ of $L$; alternatively we may write $(i, j, k) \in L$. In this latter form, the triples of any Latin square will always be specified in (row, column, entry) order. Note however that in a biembedding of two Latin squares, the vertices of faces forming one colour class will appear clockwise in the cyclic order (row, column, entry), while those forming the other will appear anticlockwise if taken in the same cyclic order. A parallel class of triples in a $\mathrm{TD}(3, n)$ is a set of triples in which each point of the design appears precisely once. Such a parallel class is equivalent to a transversal in a corresponding Latin square.

For Latin squares $A$ and $B$ of order $n$ with common sets of row labels, of column labels, and of entries, we will write $A \bowtie B$ (to be read as $A$ biembeds with $B$ without relabelling), if the particular realizations of $A$ and $B$ form an embedding in a surface; that is to say that the triangles formed by the (row, column, entry) triples of $A$ and $B$ may be sewn together along their common edges to form the surface. As a matter of terminology, we will refer to vertices as row, column or entry vertices, so that a triple $(a, b, c) \in A$ gives a face with row vertex $a$, column vertex $b$ and entry vertex $c$. In order to verify that $A \bowtie B$, it is necessary to check that the sewing operation generates a genuine surface and not a pseudosurface. This can be done by checking that the rotation at each vertex is a single cycle of length $2 n$ rather than a set of shorter cycles. With a slight abuse of notation we also use $A \bowtie B$ to denote the actual embedding itself. Furthermore, if $B$ is known to have a transversal, we will add a + sign and write $A \bowtie B+$. We will also identify a group $G$ with its Cayley table, so that we may write $G \bowtie H$, meaning that the Latin square formed by a Cayley table of $G$ biembeds with the Latin square $H$.

\section{The theorem}

Theorem 2.1 Suppose that $G$ is an Abelian group and that $G \neq C_{2}^{2}$. Then $G \bowtie H$ for some Latin square $H$. There is no $H$ for which $C_{2}^{2} \bowtie H$.

The proof of this result follows from the construction given in [4], a known result concerning regular embeddings, and four additional lemmas. We start by citing the earlier results. 
Theorem 2.2 [4] Suppose that $L \bowtie L^{\prime}$, where $L$ and $L^{\prime}$ are of order $n$ and have row, column and entry labels $\{0,1, \ldots, n-1\}$. Suppose also that $Q \bowtie Q^{\prime}+$, where $Q$ and $Q^{\prime}$ are of order $m$ and have row, column and entry labels $\{0,1, \ldots, m-1\}$, and that the square $Q^{\prime}$ has a transversal $\mathcal{T}$. Define squares $Q(L)$ and $Q^{\prime}\left(L, \mathcal{T}, L^{\prime}\right)$ by

$$
\begin{aligned}
Q(L)(n u+i, n v+j) & =n Q(u, v)+L(i, j), \\
Q^{\prime}\left(L, \mathcal{T}, L^{\prime}\right)(n u+i, n v+j) & =n Q^{\prime}(u, v)+k,
\end{aligned}
$$

for $0 \leq u, v \leq m-1$ and $0 \leq i, j \leq n-1$, where

$$
k= \begin{cases}L(i, j) & \text { if }(u, v, w) \notin \mathcal{T} \text { for any } w \\ L^{\prime}(i, j) & \text { if there exists } w \text { such that }(u, v, w) \in \mathcal{T},\end{cases}
$$

Then $Q(L)$ and $Q^{\prime}\left(L, \mathcal{T}, L^{\prime}\right)$ are Latin squares of order $m n$ with row, column and entry labels $\{0,1, \ldots, m n-1\}$, and $Q(L) \bowtie Q^{\prime}\left(L, \mathcal{T}, L^{\prime}\right)$.

The square $Q(L)$ is partitioned into $n \times n$ subsquares which are just relabelled copies of $L$. The square $Q^{\prime}\left(L, \mathcal{T}, L^{\prime}\right)$ has a similar structure but the subsquares corresponding to the transversal $\mathcal{T}$ are relabelled copies of $L^{\prime}$. Note that if $L^{\prime}$ has a transversal, then among the relabelled copies of $L^{\prime}$ one can find a transversal in $Q^{\prime}\left(L, \mathcal{T}, L^{\prime}\right)$. This feature facilitates re-application of the construction. Note also that if $Q$ and $L$ are groups then $Q(L)$ is a Cayley table for the group $Q \times L$.

As an application of Theorem 2.2, it was shown in [4] that all Abelian 2-groups, apart from $C_{2}^{2}$, appear in biembeddings.

Theorem $2.3[4]$ For every $i \geq 1, i \neq 2$, there is a Latin square $A_{i}$ such that $C_{2}^{i} \bowtie A_{i}$. Moreover, if $i>2$ then the square $A_{i}$ may be taken to contain a transversal. There is no $A_{2}$ such that $C_{2}^{2} \bowtie A_{2}$.

The next result, the present form of which is taken from [2], asserts the existence of a biembedding of each cyclic group $C_{t}$. To explain the terminology we digress slightly. We will say that an embedding of a graph $G$ is regular or flag-transitive if for every two flags, i.e. triples $\left(v_{1}, e_{1}, f_{1}\right)$ and $\left(v_{2}, e_{2}, f_{2}\right)$, where $e_{i}$ is an edge incident with the vertex $v_{i}$ and the face $f_{i}$, there exists an automorphism of the embedding which maps $v_{1}$ to $v_{2}, e_{1}$ to $e_{2}$ and $f_{1}$ to $f_{2}$. This definition of regularity requires the admission of automorphisms that reverse the orientation of an orientable surface, although some authors require global orientation to be preserved. We refer the reader to [1, p.36] for further discussion of the terminology. When viewed as a face 2-colourable triangular embedding of $K_{t, t, t}$, the biembedding described in Theorem 2.4 is regular and, as shown in [3], is (up to isomorphism) the unique biembedding of $C_{t}$ with this regularity property. Accordingly, we will refer to it as the regular biembedding of $C_{t}$.

Theorem 2.4 [2] If the Latin squares $C_{t}$ and $C_{t}^{\prime}$ are defined by $C_{t}(i, j)=i+j$ $\bmod t$ and $C_{t}^{\prime}(i, j)=i+j+1 \bmod t$, then $C_{t} \bowtie C_{t}^{\prime}$. Moreover, if $t$ is odd then $C_{t}^{\prime}$ has a transversal $\mathcal{T}=\left\{(i, i, 2 i+1): i \in \mathbb{Z}_{t}\right\}$. 
We next state and prove the four lemmas that will enable us to complete the proof of Theorem 2.1.

Lemma 2.1 If $i \geq 3$ then $C_{2^{i}} \bowtie H_{i}+$ for some $H_{i}$ having a transversal.

Proof. We deal first with the case $i=3$. It is easy to check that the square $C_{8}$ biembeds with

$H_{3}=$\begin{tabular}{c|cccccccc} 
& 0 & 1 & 2 & 3 & 4 & 5 & 6 & 7 \\
\hline 0 & 7 & 2 & 3 & 0 & 1 & 4 & 5 & 6 \\
1 & 3 & 4 & 5 & 1 & 6 & 7 & 0 & 2 \\
2 & 1 & 5 & 6 & 4 & 7 & 0 & 2 & 3 \\
3 & 6 & 3 & 7 & 5 & 0 & 2 & 4 & 1 \\
4 & 5 & 7 & 0 & 6 & 2 & 3 & 1 & 4 \\
5 & 2 & 0 & 4 & 7 & 3 & 1 & 6 & 5 \\
6 & 0 & 6 & 1 & 2 & 4 & 5 & 3 & 7 \\
7 & 4 & 1 & 2 & 3 & 5 & 6 & 7 & 0
\end{tabular}

Note that $H_{3}$ has a transversal (shown highlighted) on the leading diagonal.

For $i>3$, put $n=2^{i}$. To form $H_{i}$, start with $C_{n}^{\prime}$ as defined above but alter 16 entries to give the following triples:

$$
\begin{array}{cccc}
\left(0,0, \frac{n}{2}+1\right), & \left(0, \frac{n}{4}, \frac{3 n}{4}+1\right), & \left(0, \frac{n}{2}, 1\right), & \left(0, \frac{3 n}{4}, \frac{n}{4}+1\right), \\
\left(\frac{n}{4}, 0, \frac{3 n}{4}+1\right), & \left(\frac{n}{4}, \frac{n}{4}, 1\right), & \left(\frac{n}{4}, \frac{n}{2}, \frac{n}{4}+1\right), & \left(\frac{n}{4}, \frac{3 n}{4}, \frac{n}{2}+1\right), \\
\left(\frac{n}{2}, 0,1\right), & \left(\frac{n}{2}, \frac{n}{4}, \frac{n}{4}+1\right), & \left(\frac{n}{2}, \frac{n}{2}, \frac{n}{2}+1\right), & \left(\frac{n}{2}, \frac{3 n}{4}, \frac{3 n}{4}+1\right), \\
\left(\frac{3 n}{4}, 0, \frac{n}{4}+1\right), & \left(\frac{3 n}{4}, \frac{n}{4}, \frac{n}{2}+1\right), & \left(\frac{3 n}{4}, \frac{n}{2}, \frac{3 n}{4}+1\right), & \left(\frac{3 n}{4}, \frac{3 n}{4}, 1\right) .
\end{array}
$$

Since $C_{n} \bowtie C_{n}^{\prime}$, it follows that in the embedding of $C_{n}$ with $H_{i}$ the rotations at row vertices, other than those corresponding to rows $0, \frac{n}{4}, \frac{n}{2}$ and $\frac{3 n}{4}$, will be cycles of length $2 n$. The same goes for column vertices, while for entry vertices the only possible exceptions are for the entries $1, \frac{n}{4}+1, \frac{n}{2}+1$ and $\frac{3 n}{4}+1$. The rotation at a row vertex alternates column and entry vertices, so to prove that the rotation at the row vertex 0 is also a single cycle of length $2 n$, it suffices to list these entry vertices in the order in which they appear and to verify that they form a single cycle of length $n$. The sequence(s) of entry vertices around this row vertex is (are) determined by the following permutation given in two-line form, where the top line is row 0 of $C_{n}$ and the bottom line is row 0 of $H_{i}$.

$$
\begin{aligned}
& \left(\begin{array}{cccccccccccc}
0 & 1 & 2 & \ldots & \frac{n}{4}-1 & \frac{n}{4} & \frac{n}{4}+1 & \ldots & \frac{n}{2}-1 & \frac{n}{2} & \frac{n}{2}+1 & \ldots \\
\frac{n}{2}+1 & 2 & 3 & \ldots & \frac{n}{4} & \frac{3 n}{4}+1 & \frac{n}{4}+2 & \ldots & \frac{n}{2} & \frac{n}{2}+2 & \ldots
\end{array}\right.
\end{aligned}
$$

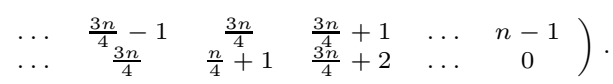

This gives

$$
\begin{aligned}
\left(1,2,3, \ldots, \frac{n}{4}-1, \frac{n}{4}, \frac{3 n}{4}+1, \frac{3 n}{4}+2, \ldots, n-1,0, \frac{n}{2}+1, \frac{n}{2}+2, \ldots\right. \\
\left.\ldots, \frac{3 n}{4}-1, \frac{3 n}{4}, \frac{n}{4}+1, \frac{n}{4}+2, \ldots, \frac{n}{2}-1, \frac{n}{2}\right)
\end{aligned}
$$


which is a single cycle of length $n$. The same cycle will be obtained for rows $\frac{n}{4}, \frac{n}{2}$ and $\frac{3 n}{4}$ because these rows of $C_{n}$ and $H_{i}$ are just cyclic shifts of row 0 by $\frac{n}{4}, \frac{n}{2}$ and $\frac{3 n}{4}$ respectively. Furthermore, the squares are symmetric, so the same cycle will be obtained for the columns labelled $0, \frac{n}{4}, \frac{n}{2}$ and $\frac{3 n}{4}$.

Finally we consider the rotations at the four exceptional entry vertices. Each such rotation alternates row and column vertices, and if the row vertices form a cycle of length $n$ then the rotation will form a single cycle of length $2 n$. We start by considering entry 1 , and constructing a permutation representing the sequence(s) of row vertices around this entry vertex. In two-line format this is given by taking the top line to be the list, in column order, of the row labels corresponding to the entry 1 in $C_{n}$, and the bottom line is formed similarly from $H_{i}$. It may help the reader for us to note that, with arithmetic modulo $n$, column $j$ gives rise to a pair of the form ${ }_{x}^{1-j}$ in the permutation. The permutation is as follows.

$$
\begin{aligned}
& \left(\begin{array}{cccccccccccc}
1 & 0 & n-1 & \ldots & \frac{3 n}{4}+2 & \frac{3 n}{4}+1 & \frac{3 n}{4} & \ldots & \frac{n}{2}+2 & \frac{n}{2}+1 & \frac{n}{2} & \ldots \\
\frac{n}{2} & n-1 & n-2 & \ldots & \frac{3 n}{4}+1 & \frac{n}{4} & \frac{3 n}{4}-1 & \ldots & \frac{n}{2}+1 & 0 & \frac{n}{2}-1 & \ldots
\end{array}\right. \\
& \left.\begin{array}{lccccc}
\ldots & \frac{n}{4}+2 & \frac{n}{4}+1 & \frac{n}{4} & \ldots & 2 \\
\cdots & \frac{n}{4}+1 & \frac{3 n}{4} & \frac{n}{4}-1 & \ldots & 1
\end{array}\right)
\end{aligned}
$$

This gives

$$
\begin{aligned}
\left(0, n-1, n-2, \ldots, \frac{3 n}{4}+2, \frac{3 n}{4}+1, \frac{n}{4}, \frac{n}{4}-1, \ldots, 2,1, \frac{n}{2}, \frac{n}{2}-1, \ldots\right. \\
\left.\ldots, \frac{n}{4}+2, \frac{n}{4}+1, \frac{3 n}{4}, \frac{3 n}{4}-1, \ldots, \frac{n}{2}+2, \frac{n}{2}+1\right)
\end{aligned}
$$

which is a single cycle of length $n$ (in fact the inverse of the row and column cycle). By the same argument as for the rows, the same cycle will be obtained for the entries $\frac{n}{4}+1, \frac{n}{2}+1$ and $\frac{3 n}{4}+1$. It therefore follows that $C_{n} \bowtie H_{i}$.

All that remains is to identify a transversal in $H_{i}$. Noting the assumption that $n \geq 16$, this is given by the triples

$$
\begin{gathered}
(x, x+2,2 x+3) \text { for } x=0,1, \ldots, \frac{n}{2}-3, \text { except for } x=\frac{n}{4}-1, \\
(x, x-1,2 x) \text { for } x=\frac{n}{2}+2, \frac{n}{2}+3, \ldots, n-1, \text { except for } x=\frac{3 n}{4}, \\
\left(\frac{n}{4}-1, \frac{3 n}{4}-1, n-1\right),\left(\frac{n}{2}-2,1, \frac{n}{2}\right),\left(\frac{n}{2}-1, \frac{n}{2}, 0\right),\left(\frac{n}{2}, 0,1\right), \\
\left(\frac{n}{2}+1, n-1, \frac{n}{2}+1\right),\left(\frac{3 n}{4}, \frac{n}{4}+1,2\right) .
\end{gathered}
$$

Lemma 2.2 If $i \geq 2$ then $C_{4}^{i} \bowtie H_{i}+$ for some $H_{i}$ having a transversal.

Proof. We show that $C_{4}^{2} \bowtie H_{2}+$ and $C_{4}^{3} \bowtie H_{3}+$ for some $H_{2}$ and $H_{3}$, each having a transversal. The result will then follow by use of Theorem 2.2 since, for $i \geq 4$, we have $C_{4}^{i}=C_{4}^{2} \times C_{4}^{2} \times \cdots \times C_{4}^{2} \times C_{4}^{j}$, where $j=2$ or 3 . We may take 


\begin{tabular}{r|rrrrrrrrrrrrrrrr} 
& 0 & 1 & 2 & 3 & 4 & 5 & 6 & 7 & 8 & 9 & 10 & 11 & 12 & 13 & 14 & 15 \\
\hline 0 & 7 & 4 & 5 & 14 & 0 & 1 & 2 & 3 & 15 & 12 & 13 & 6 & 8 & 9 & 10 & 11 \\
1 & 6 & 7 & 12 & 5 & 1 & 2 & 3 & 0 & 14 & 15 & 4 & 13 & 9 & 10 & 11 & 8 \\
2 & 5 & 6 & 7 & 4 & 10 & 3 & 0 & 1 & 13 & 14 & 15 & 12 & 2 & 11 & 8 & 9 \\
3 & 4 & 5 & 6 & 7 & 3 & 8 & 1 & 2 & 12 & 13 & 14 & 15 & 11 & 0 & 9 & 10 \\
4 & 2 & 11 & 0 & 1 & 5 & 6 & 7 & 4 & 10 & 3 & 8 & 9 & 13 & 14 & 15 & 12 \\
$H_{2}=$ & 0 & 1 & 2 & 3 & 7 & 4 & 13 & 6 & 8 & 9 & 10 & 11 & 15 & 12 & 5 & 14 \\
5 & 3 & 0 & 1 & 2 & 4 & 5 & 6 & 15 & 11 & 8 & 9 & 10 & 12 & 13 & 14 & 7 \\
7 & 9 & 2 & 3 & 0 & 6 & 7 & 4 & 5 & 1 & 10 & 11 & 8 & 14 & 15 & 12 & 13 \\
8 & 15 & 12 & 13 & 6 & 8 & 9 & 10 & 11 & 7 & 4 & 5 & 14 & 0 & 1 & 2 & 3 \\
9 & 14 & 15 & 4 & 13 & 9 & 10 & 11 & 8 & 6 & 7 & 12 & 5 & 1 & 2 & 3 & 0 \\
10 & 13 & 14 & 15 & 12 & 2 & 11 & 8 & 9 & 5 & 6 & 7 & 4 & 10 & 3 & 0 & 1 \\
11 & 12 & 13 & 14 & 15 & 11 & 0 & 9 & 10 & 4 & 5 & 6 & 7 & 3 & 8 & 1 & 2 \\
12 & 10 & 3 & 8 & 9 & 13 & 14 & 15 & 12 & 2 & 11 & 0 & 1 & 5 & 6 & 7 & 4 \\
13 & 8 & 9 & 10 & 11 & 15 & 12 & 5 & 14 & 0 & 1 & 2 & 3 & 7 & 4 & 13 & 6 \\
14 & 11 & 8 & 9 & 10 & 12 & 13 & 14 & 7 & 3 & 0 & 1 & 2 & 4 & 5 & 6 & 15 \\
15 & 1 & 10 & 11 & 8 & {$[14$} & 15 & 12 & 13 & 9 & 2 & 3 & 0 & 6 & 7 & 4 & 5
\end{tabular}

To verify that $C_{4}^{2}$ biembeds with $H_{2}$ the reader should check the rotations at each of the row, column and entry vertices. The square $\mathrm{H}_{2}$ has two disjoint transversals, one highlighted, $\mathcal{T}_{2}$, and one boxed, $\mathcal{T}_{2}^{\prime}$. The former is given by $\mathcal{T}_{2}=\{(0,3,14),(1,2,12),(2,4,10),(3,5,8),(4,1,11),(5,6,13),(6,7,15)$, $(7,0,9),(8,14,2),(9,9,7),(10,15,1),(11,8,4),(12,10,0),(13,11,3),(14,13,5)$, $(15,12,6)\}$. It follows from Theorem 2.2 that $C_{4}^{3}=C_{4}^{2}\left(C_{4}\right)$ biembeds with $H_{3}=H_{2}\left(C_{4}, \mathcal{T}_{2}, C_{4}^{\prime}\right)$. These squares are both of order 64 , and consequently, too large to display here. Nevertheless, the structure of $H_{3}$ should be clear and it has a transversal, $\mathcal{T}_{3}$, obtained from $\mathcal{T}_{2}$ and $\mathcal{T}_{2}^{\prime}$ and given by the 64 triples shown in Table 1. This completes the proof of the lemma.

$$
\begin{array}{cccc}
(0,12,57), & (1,13,59), & (2,46,24), & (3,47,26), \\
(4,8,49), & (5,9,51), & (6,42,16), & (7,43,18), \\
(8,16,41), & (9,17,43), & (10,50,8), & (11,51,10), \\
(12,20,33), & (13,21,35), & (14,54,0), & (15,55,2), \\
(16,4,45), & (17,5,47), & (18,38,12), & (19,39,14), \\
(20,24,53), & (21,25,55), & (22,58,20), & (23,59,22), \\
(24,28,61), & (25,29,63), & (26,62,28), & (27,63,30), \\
(28,0,37), & (29,1,39), & (30,34,4), & (31,35,6), \\
(32,56,9), & (33,57,11), & (34,26,40), & (35,27,42), \\
(36,36,29), & (37,37,31), & (38,6,60), & (39,7,62), \\
(40,60,5), & (41,61,7), & (42,30,36), & (43,31,38), \\
(44,32,17), & (45,33,19), & (46,2,48), & (47,3,50), \\
(48,40,1), & (49,41,3), & (50,10,32), & (51,11,34), \\
(52,44,13), & (53,45,15), & (54,14,44), & (55,15,46), \\
(56,52,21), & (57,53,23), & (58,22,52), & (59,23,54), \\
(60,48,25), & (61,49,27), & (62,18,56), & (63,19,58) .
\end{array}
$$

Table 1 . The transversal $\mathcal{T}_{3}$ in $H_{3}$. 
Lemma 2.3 If $i \geq 2$ and $n=2^{i}$, then $\left(C_{2} \times C_{n}\right) \bowtie H_{n}+$ for some $H_{n}$ having a transversal.

Proof. The proof is by direct construction. The square $H_{n}$ is a copy of the Cayley table of the dihedral group $D_{n}$. We give standard forms for the Cayley tables of $C_{2} \times C_{n}$ and $D_{n}$, and then give three permutations that are applied respectively to the entries, column labels and row labels of $D_{n}$ in order to form $H_{n}$. In the next paragraph it is shown that $D_{n}$, and hence $H_{n}$, has a transversal. Rather more tedious is the proof that the squares $C_{2} \times C_{n}$ and $H_{n}$ form a biembedding; we do this by examining the rotation at each vertex. In our squares of order $2 n$, the row labels, column labels and entries will be taken as $0,1, \ldots, n-1,0^{\prime}, 1^{\prime}, \ldots, n-1^{\prime}$, where $n-1^{\prime}$ is written for $(n-1)^{\prime}$ to save on excessive use of brackets; a similar gloss will be applied to other compound terms. All arithmetic encountered is to be taken in $\mathbb{Z}_{n}$. Our standard form for $D_{n}$ is shown in Figure 1.

\begin{tabular}{r|cccccccccc} 
& 0 & 1 & 2 & $\ldots$ & $n-1$ & $0^{\prime}$ & $1^{\prime}$ & $2^{\prime}$ & $\ldots$ & $n-1^{\prime}$ \\
\hline 0 & 0 & 1 & 2 & $\ldots$ & $n-1$ & $0^{\prime}$ & $1^{\prime}$ & $2^{\prime}$ & $\ldots$ & $n-1^{\prime}$ \\
1 & 1 & 2 & 3 & $\ldots$ & 0 & $1^{\prime}$ & $2^{\prime}$ & $3^{\prime}$ & $\ldots$ & $0^{\prime}$ \\
2 & 2 & 3 & 4 & $\ldots$ & 1 & $2^{\prime}$ & $3^{\prime}$ & $4^{\prime}$ & $\ldots$ & $1^{\prime}$ \\
$\vdots$ & & & & $\vdots$ & & & & & $\vdots$ & \\
$n-1$ & $n-1$ & 0 & 1 & $\ldots$ & $n-2$ & $n-1^{\prime}$ & $0^{\prime}$ & $1^{\prime}$ & $\ldots$ & $n-2^{\prime}$ \\
$0^{\prime}$ & $0^{\prime}$ & $n-1^{\prime}$ & $n-2^{\prime}$ & $\ldots$ & $1^{\prime}$ & 0 & $n-1$ & $n-2$ & $\ldots$ & 1 \\
$1^{\prime}$ & $1^{\prime}$ & $0^{\prime}$ & $n-1^{\prime}$ & $\ldots$ & $2^{\prime}$ & 1 & 0 & $n-1$ & $\ldots$ & 2 \\
$2^{\prime}$ & $2^{\prime}$ & $1^{\prime}$ & $0^{\prime}$ & $\ldots$ & $3^{\prime}$ & 2 & 1 & 0 & $\ldots$ & 3 \\
$\vdots$ & & & & $\vdots$ & & & & & $\vdots$ & \\
$n-1^{\prime}$ & $n-1^{\prime}$ & $n-2^{\prime}$ & $n-3^{\prime}$ & $\ldots$ & $0^{\prime}$ & $n-1$ & $n-2$ & $n-3$ & $\ldots$ & 0
\end{tabular}

Figure 1. The dihedral group $D_{n}$.

A transversal in $D_{n}$ is given by the triples

$$
\begin{gathered}
(0,0,0),(1,1,2), \ldots,\left(\frac{n}{2}-1, \frac{n}{2}-1, n-2\right), \\
\left(\frac{n}{2}, \frac{n}{2}^{\prime}, 0^{\prime}\right),\left(\frac{n}{2}+1, \frac{n}{2}+1^{\prime}, 2^{\prime}\right), \ldots,\left(n-1, n-1^{\prime}, n-2^{\prime}\right), \\
\left(0^{\prime}, n-1,1^{\prime}\right),\left(1^{\prime}, n-2,3^{\prime}\right), \ldots,\left(\frac{n}{2}-1^{\prime}, \frac{n}{2}, n-1^{\prime}\right), \\
\left(\frac{n}{2}^{\prime}, \frac{n}{2}-1^{\prime}, 1\right),\left(\frac{n}{2}+1^{\prime}, \frac{n}{2}-2^{\prime}, 3\right), \ldots,\left(n-1^{\prime}, 0^{\prime}, n-1\right) .
\end{gathered}
$$

We next apply the following permutations to $D_{n}$ to form $H_{n}$.

$$
\begin{gathered}
\text { Entries: }\left(\begin{array}{ccccccccccc}
0 & 1 & 2 & \ldots & n-2 & n-1 & 0^{\prime} & 1^{\prime} & 2^{\prime} & \ldots & n-1^{\prime} \\
n-2 & n-3 & n-4 & \ldots & 0 & n-1 & 0^{\prime} & 1^{\prime} & 2^{\prime} & \ldots & n-1^{\prime}
\end{array}\right), \\
\text { columns: }\left(\begin{array}{ccccccccccc}
0 & 1 & 2 & \ldots & n-2 & n-1 & 0^{\prime} & 1^{\prime} & 2^{\prime} & \ldots & n-1^{\prime} \\
n-1 & n-2 & n-3 & \ldots & 1 & 0 & 0^{\prime} & 1^{\prime} & 2^{\prime} & \ldots & n-1^{\prime}
\end{array}\right),
\end{gathered}
$$




$$
\begin{aligned}
& \text { rows: }\left(\begin{array}{cccccccc}
0 & 1 & 2 & \ldots & \frac{n}{2}-2 & \frac{n}{2}-1 & \frac{n}{2} & \ldots \\
n-2^{\prime} & n-3^{\prime} & n-4^{\prime} & \ldots & \frac{n^{\prime}}{2} & \frac{n}{2}-2^{\prime} & \frac{n}{2}-3^{\prime} & \ldots
\end{array}\right. \\
& \left.\begin{array}{ccccccccc}
\ldots & n-3 & n-2 & n-1 & 0^{\prime} & 1^{\prime} & 2^{\prime} & \ldots & n-1^{\prime} \\
\ldots & 0^{\prime} & n-1^{\prime} & \frac{n}{2}-1^{\prime} & n-2 & n-3 & n-4 & \ldots & n-1
\end{array}\right) .
\end{aligned}
$$

This gives $H_{n}$ as shown in Figure 2 .

\begin{tabular}{r|cccccccccc} 
& 0 & 1 & 2 & $\ldots$ & $n-1$ & $0^{\prime}$ & $1^{\prime}$ & $2^{\prime}$ & $\ldots$ & $n-1^{\prime}$ \\
\hline 0 & $n-1^{\prime}$ & $0^{\prime}$ & $1^{\prime}$ & $\ldots$ & $n-2^{\prime}$ & 0 & 1 & 2 & $\ldots$ & $n-1$ \\
1 & $n-2^{\prime}$ & $n-1^{\prime}$ & $0^{\prime}$ & $\ldots$ & $n-3^{\prime}$ & 1 & 2 & 3 & $\ldots$ & 0 \\
2 & $n-3^{\prime}$ & $n-2^{\prime}$ & $n-1^{\prime}$ & $\ldots$ & $n-4^{\prime}$ & 2 & 3 & 4 & $\ldots$ & 1 \\
$\vdots$ & & & & $\vdots$ & & & & & $\vdots$ & \\
$n-1$ & $0^{\prime}$ & $1^{\prime}$ & $2^{\prime}$ & $\ldots$ & $n-1^{\prime}$ & $n-1$ & 0 & 1 & $\ldots$ & $n-2$ \\
$0^{\prime}$ & 2 & 3 & 4 & $\ldots$ & 1 & $n-3^{\prime}$ & $n-2^{\prime}$ & $n-1^{\prime}$ & $\ldots$ & $n-4^{\prime}$ \\
$1^{\prime}$ & 3 & 4 & 5 & $\ldots$ & 2 & $n-4^{\prime}$ & $n-3^{\prime}$ & $n-2^{\prime}$ & $\ldots$ & $n-5^{\prime}$ \\
$2^{\prime}$ & 4 & 5 & 6 & $\ldots$ & 3 & $n-5^{\prime}$ & $n-4^{\prime}$ & $n-3^{\prime}$ & $\ldots$ & $n-6^{\prime}$ \\
$\vdots$ & & & & $\vdots$ & & & & & $\vdots$ & \\
$\frac{n}{2}-2^{\prime}$ & $\frac{n}{2}$ & $\frac{n}{2}+1$ & $\frac{n}{2}+2$ & $\ldots$ & $\frac{n}{2}-1$ & $\frac{n}{2}-1^{\prime}$ & $\frac{n}{2}$ & $\frac{n}{2}+1^{\prime}$ & $\ldots$ & $\frac{n}{2}-2^{\prime}$ \\
$\frac{n}{2}-1^{\prime}$ & 0 & 1 & 2 & $\ldots$ & $n-1$ & $n-1^{\prime}$ & $0^{\prime}$ & $1^{\prime}$ & $\ldots$ & $n-2^{\prime}$ \\
$\frac{n}{2}$ & $\frac{n}{2}+1$ & $\frac{n}{2}+2$ & $\frac{n}{2}+3$ & $\ldots$ & $\frac{n}{2}$ & $\frac{n}{2}-2^{\prime}$ & $\frac{n}{2}-1^{\prime}$ & $\frac{n}{2}{ }^{\prime}$ & $\ldots$ & $\frac{n}{2}-3^{\prime}$ \\
$\frac{n}{2}+1^{\prime}$ & $\frac{n}{2}+2$ & $\frac{n}{2}+3$ & $\frac{n}{2}+4$ & $\ldots$ & $\frac{n}{2}+1$ & $\frac{n}{2}-3^{\prime}$ & $\frac{n}{2}-2^{\prime}$ & $\frac{n}{2}-1^{\prime}$ & $\ldots$ & $\frac{n}{2}-4^{\prime}$ \\
$\frac{n}{2}+2^{\prime}$ & $\frac{n}{2}+3$ & $\frac{n}{2}+4$ & $\frac{n}{2}+5$ & $\ldots$ & $\frac{n}{2}+2$ & $\frac{n}{2}-4^{\prime}$ & $\frac{n}{2}-3^{\prime}$ & $\frac{n}{2}-2^{\prime}$ & $\ldots$ & $\frac{n}{2}-5^{\prime}$ \\
$\vdots$ & & & & $\vdots$ & & & & & $\vdots$ & \\
$n-2^{\prime}$ & $n-1$ & 0 & 1 & $\ldots$ & $n-2$ & $0^{\prime}$ & $1^{\prime}$ & $2^{\prime}$ & $\ldots$ & $n-1^{\prime}$ \\
$n-1^{\prime}$ & 1 & 2 & 3 & $\ldots$ & 0 & $n-2^{\prime}$ & $n-1^{\prime}$ & $0^{\prime}$ & $\ldots$ & $n-3^{\prime}$
\end{tabular}

Figure 2. The Latin square $H_{n}$.

Our standard form for $C_{2} \times C_{n}$ is shown in Figure 3 .

\begin{tabular}{r|cccccccccc} 
& 0 & 1 & 2 & $\ldots$ & $n-1$ & $0^{\prime}$ & $1^{\prime}$ & $2^{\prime}$ & $\ldots$ & $n-1^{\prime}$ \\
\hline 0 & 0 & 1 & 2 & $\ldots$ & $n-1$ & $0^{\prime}$ & $1^{\prime}$ & $2^{\prime}$ & $\ldots$ & $n-1^{\prime}$ \\
1 & 1 & 2 & 3 & $\ldots$ & 0 & $1^{\prime}$ & $2^{\prime}$ & $3^{\prime}$ & $\ldots$ & $0^{\prime}$ \\
2 & 2 & 3 & 4 & $\ldots$ & 1 & $2^{\prime}$ & $3^{\prime}$ & $4^{\prime}$ & $\ldots$ & $1^{\prime}$ \\
$\vdots$ & & & & $\vdots$ & & & & & $\vdots$ & \\
$n-1$ & $n-1$ & 0 & 1 & $\ldots$ & $n-2$ & $n-1^{\prime}$ & $0^{\prime}$ & $1^{\prime}$ & $\ldots$ & $n-2^{\prime}$ \\
$0^{\prime}$ & $0^{\prime}$ & $1^{\prime}$ & $2^{\prime}$ & $\ldots$ & $n-1^{\prime}$ & 0 & 1 & 2 & $\ldots$ & $n-1$ \\
$1^{\prime}$ & $1^{\prime}$ & $2^{\prime}$ & $3^{\prime}$ & $\ldots$ & $0^{\prime}$ & 1 & 2 & 3 & $\ldots$ & 0 \\
$2^{\prime}$ & $2^{\prime}$ & $3^{\prime}$ & $4^{\prime}$ & $\ldots$ & $1^{\prime}$ & 2 & 3 & 4 & $\ldots$ & 1 \\
$\vdots$ & & & & $\vdots$ & & & & & $\vdots$ & \\
$n-1^{\prime}$ & $n-1^{\prime}$ & $0^{\prime}$ & $1^{\prime}$ & $\ldots$ & $n-2^{\prime}$ & $n-1$ & 0 & 1 & $\ldots$ & $n-2$
\end{tabular}

Figure 3. The group $C_{2} \times C_{n}$. 
Using Figures 2 and 3, we can construct the rotations at each vertex of the embedding. The rotation at a row vertex alternates column and entry vertices, so to prove that it is a single cycle of length $4 n$, it suffices to list the entry vertices and to verify that these form a single cycle of length $2 n$. So, consider first the rotation at the row vertex $i$ where $0 \leq i \leq n-1$. The sequence(s) of entry vertices around this row vertex is (are) determined by the following permutation given in two-line form, where the top line is the $i^{\text {th }}$ row of $C_{2} \times C_{n}$ and the bottom line is the $i^{\text {th }}$ row of $H_{n}$.

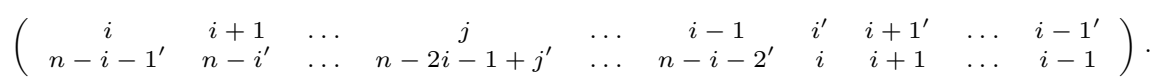

This gives $\left(i,-i-1^{\prime},-i-1,-3 i-2^{\prime},-3 i-2, \ldots,-(2 r-1) i-r, \ldots\right)$. To see that this is a cycle of length $2 n$, consider the general undashed or unprimed term (i.e. term without ') which is $-(2 r-1) i-r$. This reduces to $i$ modulo $n$ if and only if $(2 i+1) r=0$ in $\mathbb{Z}_{n}$, and this requires that $r \equiv 0(\bmod n)$. Hence the undashed terms form a cycle of length $n$ and the entire permutation is a single cycle of length $2 n$.

Next consider the rotation at a row vertex $i^{\prime}$ where $0 \leq i \leq \frac{n}{2}-2$. The corresponding sequence(s) of entry vertices around this row vertex is (are) given by

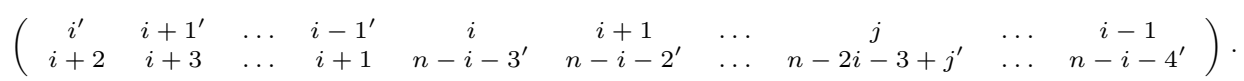

This gives $\left(i,-i-3^{\prime},-i-1,-3 i-4^{\prime},-3 i-2, \ldots,-(2 r-1) i-r, \ldots\right)$, which again forms a single cycle of length $2 n$. The same argument applies to a row vertex $i^{\prime}$ where $\frac{n}{2} \leq i \leq n-2$. It remains to consider rows $\frac{n}{2}-1^{\prime}$ and $n-1^{\prime}$. Corresponding to the former is the permutation

$$
\left(\begin{array}{cccccccc}
\frac{n}{2}-1^{\prime} & \frac{n}{2}{ }^{\prime} & \ldots & \frac{n}{2}-2^{\prime} & \frac{n}{2}-1 & \frac{n}{2} & \ldots & \frac{n}{2}-2 \\
0 & 1 & \ldots & n-1 & n-1^{\prime} & 0^{\prime} & \ldots & n-2^{\prime}
\end{array}\right) .
$$

This reduces to the single cycle $\left(0, \frac{n}{2}^{\prime}, 1, \frac{n}{2}+1^{\prime}, 2, \ldots\right)$. For row $n-1^{\prime}$ we have the permutation

$$
\left(\begin{array}{cccccccc}
n-1^{\prime} & 0^{\prime} & \ldots & n-2^{\prime} & n-1 & 0 & \ldots & n-2 \\
1 & 2 & \ldots & 0 & n-2^{\prime} & n-1^{\prime} & \ldots & n-3^{\prime}
\end{array}\right) .
$$

This reduces to the single cycle $\left(0,-1^{\prime}, 1,0^{\prime}, 2, \ldots\right)$. Thus the rotation at each row vertex is a single cycle of length $4 n$.

Next we consider the rotations at the column vertices. Note first that the columns of both $C_{2} \times C_{n}$ and $H_{n}$ have a cyclic pattern of order $n$. To be precise, for $1 \leq i \leq n-1$, column $i\left(i^{\prime}\right)$ of both $C_{2} \times C_{n}$ and $H_{n}$ may be obtained by adding $i$ to the entries in column $0\left(0^{\prime}\right)$. It therefore suffices to show that the rotations at column vertices 0 and $0^{\prime}$ are single cycles. Again we give the sequence(s) of entry vertices around column vertices 0 and $0^{\prime}$ by means of permutations in two-line form. For column vertex 0 this permutation is 
$\left(\begin{array}{ccccccccccccc}0 & 1 & \ldots & n-1 & 0^{\prime} & 1^{\prime} & \ldots & \frac{n}{2}-2^{\prime} & \frac{n}{2}-1^{\prime} & \frac{n}{2}^{\prime} & \ldots & n-2^{\prime} & n-1^{\prime} \\ n-1^{\prime} & n-2^{\prime} & \ldots & 0^{\prime} & 2 & 3 & \ldots & \frac{n}{2} & 0 & \frac{n^{2}}{2}+1 & \ldots & n-1 & 1\end{array}\right)$.

For $n=4$ the reader can easily check that this is a single cycle. In general, it gives

$\left(0,-1^{\prime}, 1,-2^{\prime},-1,0^{\prime}, 2,-3^{\prime},-2,1^{\prime}, 3,-4^{\prime},-3,2^{\prime}, 4, \ldots, \frac{n}{2}-1, \frac{n}{2}^{\prime}, \frac{n}{2}+1, \frac{n}{2}-2^{\prime}, \frac{n}{2}, \frac{n}{2}-1^{\prime}\right)$,

where sufficient terms are listed for the pattern to be apparent, and this is a cycle of length $2 n$. For column $0^{\prime}$, the permutation is

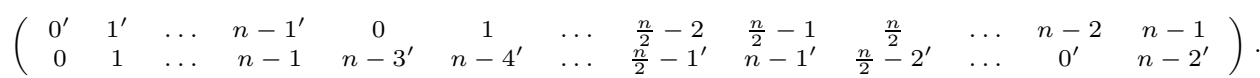

The case $n=4$ is easily checked and the general pattern is given by

$$
\left(0,-3^{\prime},-3,1^{\prime}, 1,-4^{\prime},-4,2^{\prime}, \ldots, \frac{n}{2}, \frac{n}{2}-2^{\prime}, \frac{n}{2}-2, \frac{n}{2}-1^{\prime}, \frac{n}{2}-1,-1^{\prime},-1,-2^{\prime},-2,0^{\prime}\right),
$$

which is again a cycle of length $2 n$. Thus the rotation at each column vertex is a single cycle of length $4 n$.

Finally we consider the rotations at the entry vertices. Each such rotation alternates row and column vertices, and if the row vertices form a cycle of length $2 n$ then the rotation will form a single cycle of length $4 n$. We start by considering entry vertex 0 and constructing a permutation representing the sequence(s) of row vertices around this entry vertex. In two-line format this is given by taking the top line to be the list, in column order, of the row labels corresponding to the entry 0 in $C_{2} \times C_{n}$, and the bottom line is formed similarly from $H_{n}$. The permutation is as follows.

$$
\left(\begin{array}{ccccccccccc}
0 & n-1 & n-2 & \ldots & \frac{n}{2}+1 & \frac{n}{2} & \frac{n}{2}-1 & \ldots & & \\
\frac{n}{2}-1^{\prime} & n-2^{\prime} & n-3^{\prime} & \ldots & \frac{n}{2}^{\prime} & \frac{n}{2}-2^{\prime} & \frac{n}{2}-3^{\prime} & \ldots & & & \\
& & & \ldots & 2 & 1 & 0^{\prime} & n-1^{\prime} & n-2^{\prime} & \ldots & 1^{\prime} \\
& & & \ldots & 0^{\prime} & n-1^{\prime} & 0 & n-1 & n-2 & \ldots & 1
\end{array}\right) .
$$

Again $n=4$ is easily checked, and in general this reduces to

$$
\begin{array}{r}
\left(0, \frac{n}{2}-1^{\prime}, \frac{n}{2}-1, \frac{n}{2}-3^{\prime}, \frac{n}{2}-3, \ldots, 3^{\prime}, 3,1^{\prime}, 1,-1^{\prime},-1,-2^{\prime},-2,-3^{\prime}, \ldots\right. \\
\left.\ldots, \frac{n}{2}+1, \frac{n}{2}^{\prime}, \frac{n}{2}, \frac{n}{2}-2^{\prime}, \frac{n}{2}-2, \ldots, 2^{\prime}, 2,0^{\prime}\right),
\end{array}
$$

which is a cycle of length $2 n$. Noting the cyclic pattern of the columns of the two Latin squares and to which we have already referred, it follows that exactly the same permutation will be found for each entry $i$ with $0 \leq i \leq n-1$. Turning attention to the entry vertex $0^{\prime}$ in a similar fashion, the case $n=4$ is easily checked, and for $n \geq 8$ we obtain the permutation

$$
\left.\begin{array}{ccccccccccc}
0^{\prime} & n-1^{\prime} & n-2^{\prime} & \ldots & 1^{\prime} & 0 & n-1 & n-2 & n-3 & n-4 & \ldots \\
n-1 & 0 & 1 & \ldots & n-2 & n-2^{\prime} & \frac{n}{2}-1^{\prime} & n-1^{\prime} & 0^{\prime} & 1^{\prime} & \ldots \\
& & & \ldots & \frac{n}{2}+1 & \frac{n}{2} & \frac{n}{2}-1 & \frac{n}{2}-2 & \ldots & 2 & 1 \\
& & & \ldots & \frac{n}{2}-4^{\prime} & \frac{n}{2}-3^{\prime} & \frac{n}{2}-2^{\prime} & \frac{n^{\prime}}{2} & \ldots & n-4^{\prime} & n-3^{\prime}
\end{array}\right) .
$$

This reduces to 


$$
\begin{aligned}
\left(0,-2^{\prime}, 1,-3^{\prime}, 2, \ldots, \frac{n}{2}-2, \frac{n}{2}^{\prime}, \frac{n}{2}-1, \frac{n}{2}-2^{\prime}, \frac{n}{2}+1, \frac{n}{2}-4^{\prime}, \frac{n}{2}+3, \ldots\right. \\
\left.\ldots,-3,0^{\prime},-1, \frac{n}{2}-1^{\prime}, \frac{n}{2}, \frac{n}{2}-3^{\prime}, \frac{n}{2}+2, \frac{n}{2}-5^{\prime}, \frac{n}{2}+4, \ldots,-2,-1^{\prime}\right),
\end{aligned}
$$

which is a cycle of length $2 n$. The same permutation will be found for each entry $i^{\prime}$ with $0 \leq i \leq n-1$. Thus the rotation at each entry vertex is a single cycle of length $4 n$.

We remark that for $n=2^{i}(i \geq 2)$, Lemma 2.3 also provides a biembedding of the dihedral group $D_{n}$ with a copy of $C_{2} \times C_{n}$, and it is easily seen that this latter square also has a transversal.

Lemma 2.4 If $t \geq 3$ is odd then $C_{2 t} \bowtie H_{t}+$ for some $H_{t}$ having a transversal.

Proof. Note that, as groups, $C_{2 t}=C_{2} \times C_{t}$. The regular biembedding of $C_{t}$ is $C_{t} \bowtie C_{t}^{\prime}$, where $C_{t}$ and $C_{t}^{\prime}$ are as given in Theorem 2.4. Since $t$ is odd, $C_{t}^{\prime}$ has a transversal $\mathcal{T}=\left\{(i, i, 2 i+1): i \in \mathbb{Z}_{t}\right\}$. Now apply Theorem 2.2 to form the embedding $C_{t}\left(C_{2}\right) \bowtie C_{t}^{\prime}\left(C_{2}, \mathcal{T}, C_{2}^{\prime}\right)$. But $C_{t}\left(C_{2}\right)$ is just a Cayley table for $C_{2 t}$, and so the result will follow once it is shown that $C_{t}^{\prime}\left(C_{2}, \mathcal{T}, C_{2}^{\prime}\right)$ has a transversal. This Latin square has the general form shown in Figure 4. It has a transversal $\left\{(2 i, 2 i+1,4 i+2),(2 i+1,2 i+2,4 i+5): i \in \mathbb{Z}_{t}\right\}$ which is shown highlighted in the Figure.

\begin{tabular}{r|ccccccccc} 
& 0 & 1 & 2 & 3 & 4 & 5 & $\ldots$ & $2 t-2$ & $2 t-1$ \\
\hline 0 & 3 & 2 & 4 & 5 & 6 & 7 & $\ldots$ & 0 & 1 \\
1 & 2 & 3 & 5 & 4 & 7 & 6 & $\ldots$ & 1 & 0 \\
2 & 4 & 5 & 7 & 6 & 8 & 9 & $\ldots$ & 2 & 3 \\
3 & 5 & 4 & 6 & 7 & 9 & 8 & $\ldots$ & 3 & 2 \\
4 & 6 & 7 & 8 & 9 & 11 & 10 & $\ldots$ & 4 & 5 \\
5 & 7 & 6 & 9 & 8 & 10 & 11 & $\ldots$ & 5 & 4 \\
$\vdots$ & & & & & & & $\vdots$ & & \\
$2 t-2$ & 0 & 1 & 2 & 3 & 4 & 5 & $\ldots$ & $2 t-1$ & $2 t-2$ \\
$2 t-1$ & 1 & 0 & 3 & 2 & 5 & 4 & $\ldots$ & $2 t-2$ & $2 t-1$
\end{tabular}

Figure 4. The Latin square $C_{t}^{\prime}\left(C_{2}, \mathcal{T}, C_{2}^{\prime}\right)$.

Proof of Theorem 2.1. Suppose that $G$ is an Abelian group. In general, we may write $G$ as a direct product of cyclic groups in the form

$$
G=C_{2^{i_{1}}}^{j_{1}} \times C_{2^{i_{2}}}^{j_{2}} \times \cdots \times C_{2^{i_{m}}}^{j_{m}} \times C_{k_{1}}^{l_{1}} \times C_{k_{2}}^{l_{2}} \times \cdots \times C_{k_{n}}^{l_{n}},
$$

where each $i_{s}, j_{s}$ and $l_{s}$ is a positive integer, and each $k_{s}$ is an odd positive integer. Without loss of generality we may assume that $i_{1}<i_{2}<\ldots<i_{m}$ and $k_{1}<k_{2}<\ldots<k_{n}$. If $G$ has no factor $C_{2^{i}}$, that is if $m=0$, then starting with the regular biembedding of each $C_{k_{s}}$ and applying Theorem 2.2 repeatedly, we have $G \bowtie H+$ for some $H$. In view of Lemma 2.1, the same is true if $G$ has factors $C_{2^{i}}$ for $i \geq 3$ but no factors $C_{2}$ or $C_{4}$. It remains to deal with the cases when $G$ has factors $C_{2}$ and/or $C_{4}$. 
Consider first the case when $G$ has no factors apart from $C_{2}$ and $C_{4}$, that is $G=C_{2}^{j_{1}} \times C_{4}^{j_{2}}$. If $\left(j_{1}, j_{2}\right)=(0,0)$ there is nothing to prove. Other cases are dealt with in Table 2, where $\mathrm{R}$ denotes use of a regular biembedding (Theorem 2.4), $\mathrm{L}$ a lemma, and $\mathrm{T}$ a theorem.

\begin{tabular}{|c|c|l|l|}
\hline$j_{1}$ & $j_{2}$ & $G$ & $G \bowtie H(?)$ \\
\hline 0 & 1 & $C_{4}$ & $\mathrm{R}$. \\
& $\geq 2$ & $C_{4}^{j_{2}}$ & $\mathrm{~L} 2.2$. \\
\hline 1 & 0 & $C_{2}$ & $\mathrm{R}$. \\
& 1 & $C_{2} \times C_{4}$ & $\mathrm{~L} 2.3$. \\
& 2 & $C_{4} \times\left(C_{2} \times C_{4}\right)$ & $\mathrm{R}, \mathrm{L} 2.3, \mathrm{~T} 2.2$. \\
& $\geq 3$ & $C_{4}^{j_{2}-1} \times\left(C_{2} \times C_{4}\right)$ & $\mathrm{L} 2.2, \mathrm{~L} 2.3, \mathrm{~T} 2.2$. \\
\hline 2 & 0 & $C_{2}^{2}$ & $\mathrm{~N}$ biembedding $(\mathrm{T} 2.3)$. \\
& 1 & $C_{2} \times\left(C_{2} \times C_{4}\right)$ & $\mathrm{L} 2.3, \mathrm{~T} 2.2$. \\
& 2 & $\left(C_{2} \times C_{4}\right) \times\left(C_{2} \times C_{4}\right)$ & $\mathrm{L} 2.3, \mathrm{~T} 2.2$. \\
& 3 & $C_{4} \times\left(C_{2} \times C_{4}\right) \times\left(C_{2} \times C_{4}\right)$ & $\mathrm{R}, \mathrm{L} 2.3, \mathrm{~T} 2.2$. \\
& $\geq 4$ & $C_{4}^{j_{2}}-2 \times\left(C_{2} \times C_{4}\right) \times\left(C_{2} \times C_{4}\right)$ & $\mathrm{L} 2.2, \mathrm{~L} 2.3, \mathrm{~T} 2.2$. \\
\hline$\geq 3$ & 0 & $C_{2}^{j_{1}}$ & $\mathrm{~T} 2.3$. \\
& 1 & $C_{4} \times C_{2}^{j_{1}}$ & $\mathrm{R}, \mathrm{T} 2.3, \mathrm{~T} 2.2$. \\
& $\geq 2$ & $C_{4}^{j_{2}} \times C_{2}^{j_{1}}$ & $\mathrm{~L} 2.2, \mathrm{~T} 2.3, \mathrm{~T} 2.2$. \\
\hline
\end{tabular}

Table 2. $G=C_{2}^{j_{1}} \times C_{4}^{j_{2}}$.

Next consider the case when $G=C_{2}^{j_{1}} \times C_{4}^{j_{2}} \times G^{*}$ where $G^{*}$ is non-trivial but has no factors $C_{2}$ or $C_{4}$. We already have $G^{*} \bowtie H^{*}+$ for some $H^{*}$, so if $\left(j_{1}, j_{2}\right) \neq(2,0)$, by using Theorem 2.2 and the appropriate case from Table 2 , we have $G \bowtie H$ for some $H$. All that remains to consider is the case $C_{2}^{2} \times G^{*}$. Since $G^{*}$ is non-trivial, it has a factor $C_{t}$ where $t \geq 3$ is either odd or a power of 2 , not 2 or 4 , so that $G^{*}=C_{t} \times \bar{G}$, where $\bar{G}$ may or may not be trivial. Since $\bar{G}$ has no factor $C_{2}$ or $C_{4}$, if $\bar{G}$ is non-trivial then $\bar{G} \bowtie \bar{H}+$ for some $\bar{H}$. In any case, we may write $G=C_{2} \times\left(C_{2} \times C_{t}\right) \times \bar{G}$. If $t=2^{i}$ with $i \geq 3$, then apply Lemma 2.3 and Theorem 2.2. If $t$ is odd, apply Lemma 2.4 and Theorem 2.2. This completes the proof of Theorem 2.1.

Acknowledgements Part of this work was done while the first author was visiting the Department of Mathematics at the Slovak University of Technology and he thanks the Department and the University for their hospitality. The second author acknowledges partial support by Slovak research grants VEGA 1/0489/08, APVT-20-000704 and APVV-0040-06. 


\section{References}

[1] D. Archdeacon, Topological graph theory - a survey, Congr. Numer. 115 (1996), 5-54.

[2] M. J. Grannell, T. S. Griggs and M. Knor, Biembeddings of Latin squares and Hamiltonian decompositions, Glasgow Math. J. 46 (2004), 443-457.

[3] M. J. Grannell, T. S. Griggs, M. Knor and J. Širáň, Triangulations of orientable surfaces by complete tripartite graphs, Discrete Math. 306 (2006), 600-606.

[4] M. J. Grannell, T. S. Griggs and M. Knor, On biembeddings of Latin squares, submitted.

[5] J. L. Gross and T. W. Tucker, Topological Graph Theory, John Wiley, New York (1987).

[6] G. Ringel, Map color theorem, Springer-Verlag, New York and Berlin (1974). 